\title{
An open study of pentoxyfylline and thalidomide as adjuvant therapy in the treatment of rheumatoid arthritis
}

University Hospital Leiden, The Department of Rheumatology $T$ W J Huizing C L Verweij

F C Breedveld

Department of Medical Statistics

E A van der Velde

Academic Hospital Free University, Amsterdam, The Netherlands Department of Rheumatology B A C Dijkmans

Central Laboratory of the Blood Transfusion Services, Amsterdam, The Netherlands T C T M van de Pouw Kraan

Correspondence to: T W J Huizinga, University Hospital, Department of heumatology, Building 1 $4-R, P O$ Box 9600,2300 RC Leiden, The Netherlands

Tom W J Huizinga, Ben A C Dijkmans, Edo A van der Velde, Tineke C T M van de Pouw Kraan, Cornelis L Verweij, Ferdinand C Breedveld

\begin{abstract}
Objective-Dysregulation of tumour necrosis factor $\alpha$ (TNF $\alpha$ ) production is thought to be important in rheumatoid arthritis. Since pentoxifylline and thalidomide inhibit endotoxin induced TNF production in vitro, these drugs were tested in an open study in rheumatoid arthritis patients to assess toxicity, the effect on TNF production, and the antiarthritic effects.

Methods-12 patients with active rheumatoid arthritis were treated with $1200 \mathrm{mg}$ pentoxifylline and $100 \mathrm{mg}$ thalidomide a day during 12 weeks. In addition, TNF production was assessed by ex vivo whole blood cultures stimulated with endotoxin. Results-Adverse events such as xerostomia, drowsiness, and constipation occurred in almost all patients, which led to discontinuation in three. The drugs halved the TNF production capacity during treatment (ANOVA, $P<0.03$ ) whereas production capacity of interleukin (IL) $6, I L-10$, and IL-12 was not affected. Of the nine patients who completed the study, five fulfilled the ACR $-20 \%$ response criteria after 12 weeks of treatment.

Conclusions-Although pentoxifylline/thalidomide reduced the production capacity of TNF, the benefit/side effects ratio was poor due to multiple adverse effects, while clinical observation suggests limited efficacy.
\end{abstract}

(Ann Rheum Dis 1996;55:833-836)

The identification of tumour necrosis factor $\alpha$ (TNF $\alpha$ ) as one of the key mediators of inflammation in rheumatoid arthritis has led to randomised trials using anti-TNF $\alpha$ monoclonal antibody (Mab) which were reported to have beneficial results. ${ }^{12}$ These results support the hypothesis that reducing TNF concentrations is an attractive goal in the treatment of rheumatoid arthritis.

TNF is an inducible cytokine that is not produced in resting cells. Stimulation of monocytes by endotoxin is used as a model system to select drugs that inhibit TNF production. Pentoxifylline, a methylxanthine derivative and phosphodiesterase inhibitor, reduces TNF production in cultured monocytes by inhibition of transcription of the TNF $\alpha$ gene. $^{3}$ In patients, pentoxifylline reduced clinical indices of inflammation in open studies in various disease states such as bone marrow transplantation, ${ }^{4}$ AIDS, ${ }^{5}$ and cerebral malaria. ${ }^{6}$ In an open study in rheumatoid arthritis patients, Maksymowych et al ${ }^{7}$ showed that pentoxifylline has both a moderately beneficial clinical response and an inhibitory effect on TNF production capacity.

Thalidomide is a drug that selectively inhibits TNF production by monocytes, presumably by enhancing degradation of messanger RNA (mRNA) ${ }^{89}$ In various groups of patients, for example those suffering from discoid lupus erythematosus, prurigo nodularis, recurrent aphthosis, Jessner's lymphocytic infiltration, pulmonary tuberculosis, ${ }^{10}$ chronic graft versus host disease, ${ }^{11}$ and rheumatoid arthritis, ${ }^{12}$ open studies have been performed in which thalidomide reduced clinical indices of inflammation. In severe aphthosis the beneficial effects of thalidomide were confirmed in a placebo controlled study. ${ }^{13}$

The mechanisms underlying the inhibition of TNF $\alpha$ production by pentoxifylline and thalidomide are theoretically synergistic. We therefore carried out an open trial in rheumatoid arthritis patients to determine whether the combination of pentoxifylline and thalidomide was toxic, effective with regard to reduction of the endotoxin induced TNF production ex vivo, and had an antiarthritic effect.

\section{Methods}

STUDY DESIGN

Patients received pentoxifylline $400 \mathrm{mg}$ three times daily and $100 \mathrm{mg}$ thalidomide once a day in an open study over 12 weeks. During the study, existing antirheumatic drug treatment was continued.

PATIENT POPULATION

Twelve patients with rheumatoid arthritis according to the 1987 criteria of the American Rheumatism Association were recruited from the outpatient clinic of the Academic Hospital Leiden, The Netherlands. Patients were included if they met the inclusion criteria for active disease, defined by the presence of six or more swollen joints (out of 28) and at least two of the following criteria: (1) the presence of nine or more joints that are painful on motion or tender on pressure; (2) morning stiffness lasting at least 45 minutes; (3) an erythrocyte sedimentation rate (ESR) of at least $28 \mathrm{~mm}$ during the first hour. Exclusion criteria were a history of peripheral sensory neuropathy, 
Table 1 Changes in production of cytokines in whole blood cultures

\begin{tabular}{|c|c|c|c|c|c|c|}
\hline & \multicolumn{5}{|l|}{ Week No } & \multirow{2}{*}{ ANOVA test $P$} \\
\hline & 0 & 4 & 8 & 12 & 16 & \\
\hline Stimulus $100 \mathrm{ng}$ endotoxin TNF (measured by ELISA) & $9.1(7)$ & $4(5)$ & $4.1(4.6)$ & $11(7)$ & $10(5)$ & 0.005 \\
\hline Stimulus $1000 \mathrm{ng}$ endotoxin TNF (measured by ELISA) & $9.8(8)$ & $5.3(6.4)$ & $8.2(13.3)$ & $16.5(9.9)$ & $14.2(9.8)$ & 0.011 \\
\hline TNF (measured by bioassay) & $21(3)$ & $14.8(5.4)$ & $16.4(16.7)$ & $26.1(3.8)$ & $22(3.3)$ & 0.00 \\
\hline IL6 (measured by ELISA) & 39 (15) & $30(18)$ & $28(23)$ & $33(17)$ & $32(17)$ & 0.8 \\
\hline IL10 (measured by ELISA) & $7.5(4.3)$ & $8.1(5.4)$ & $9.2(6.6)$ & $12.5(7.8)$ & $8.7(7.6)$ & 0.37 \\
\hline IL12 (p40 subunit measured by ELISA) & $12.5(4.7)$ & $11.6(10)$ & $10(8)$ & $10.3(8.8)$ & $12.8(6.4)$ & 0.7 \\
\hline
\end{tabular}

active gastroduodenal ulcer, severe constipation, vertigo of vestibular disease, females with childbearing potential, or a change in the antirheumatic treatment during the preceding three months.

\section{EVALUATION}

Patients

Clinical and laboratory assessments were performed at the start of the study, at week 4 and 8 during the study, at week 12 one day after the study, and 4 weeks after discontinuation of the treatment. The following clinical indices were assessed:

(1) The number of swollen joints (out of 28)

(2) The number of tender joints

(3) The number of joints with restricted motion

(4) The grip strength using a dynamometer

(5) The physician's and the patient's global assessment of disease activity on a scale of 0-10.

Patients were always evaluated between 9 and 11 am and phlebotomy was performed at that time. Patients were also evaluated as responders or non-responders according to the modified American College of Rheumatology (ACR) core set of improvements.

\section{Cytokine production}

TNF $\alpha$, interleukin (IL) 10, IL-12, and IL-6 production capacity was evaluated using a whole blood stimulation assay as described. The concentration of TNF was determined by enzyme linked immunosorbent assay (ELISA) and by bioassay. All samples were determined in duplicate. The concentration of IL-10 was determined by ELISA using a capture anti-IL-10 monoclonal antibody (JES3-9D7, Pharmingen, San Diego, CA, USA) and a biotinylated antibody for detection (JES3-1268, Pharmingen). IL-6 was determined by a sandwich ELISA obtained from Prof Aarden (Central Laboratory of the Blood Transfusion Services, Amsterdam, The Netherlands). IL-12 p40 subunit was determined by ELISA.

DATA ANALYSIS AND STATISTICS

Patients were analysed on the basis of completers. The correlation between the decrease in the amount of swollen joints and the decrease of TNF production capacity was calculated with the reduction in TNF production capacity (endotoxin induced TNF production on week 0 and week 16 divided by 2 minus endotoxin induced TNF production during treatment at week 4 and 8 divided by two) and the reduction of swollen joints (number of swollen joints on week 0 and week 16 divided by 2 minus number of swollen joints on week 4 and week 8 divided by 2). Data were analysed with an analysis of variance (ANOVA) for repeated measures.

\section{Results}

TOXICITY OF PENTOXIFYLLINE/THALIDOMIDE

Twelve patients were enrolled in the study. The average age was 64.2 (SD 7.8) years and the disease duration 10.1 (5.4) years. All patients were rheumatoid factor positive and suffered from erosive disease. The number of disease modifying antirheumatic drugs previously used was 3.1 (1.5). The present medication was continued during the study and consisted of a disease modifying antirheumatic drug and a non-steroidal anti-inflammatory drug. Two patients also received low dose steroids. One patient developed a rash after one week of pentoxifylline/thalidomide which disappeared after the experimental medication was stopped. Two patients left the study because of severe constipation after one and four weeks, respectively. These patients were followed up on all scheduled visits. Side effects asked about from a pre-established list were xerostomia $33 \%$ (before treatment) and $100 \%$ (ever during treatment); drowsiness $33 \%$ and $77 \%$; constipation $22 \%$ and $77 \%$; oedema $44 \%$ and $66 \%$; dizziness $11 \%$ and $33 \%$.

\section{TNF PRODUCTION CAPACITY AND PENTOXIFYLLINE/} THALIDOMIDE

Whole blood cultures in the presence of endotoxin were performed to measure inhibition of production of TNF ex vivo. Whole blood cultures may yield variable results in one healthy person tested over time. Therefore one control person was tested on 24 different occasions. The mean amount of TNF produced upon culture with $100 \mathrm{ng}$ endotoxin was 14.7 (SD 5.0) $\mathrm{ng} \mathrm{ml}^{-1}$ (range 7-23) and upon culture with $1000 \mathrm{ng}$ endotoxin was $20.3(5.9) \mathrm{ng} \mathrm{ml}^{-1}$ (range 9-32). In order to detect changes despite this variability, blood was obtained before the medication, two times during medication, one day after cessation of medication, and four weeks after discontinuation of the medication. Table 1 shows that the amount of TNF produced was reduced significantly during treatment with pentoxifylline/ thalidomide ( $P=0.01$, ANOVA). The amount of TNF produced in the whole blood cultures before the study (at week 0 ) was less than after the study but the change was not significant $(P=$ 0.33). If TNF was measured by a bioassay instead of an ELISA, inhibition of TNF production was also found (ANOVA, $P=0.037$ ) (table 1). 
Table 2 Clinical variables. Baseline values are number of joints out of 28. Health assessment questionnaire (HAQ) has a maximum value of 3. Global assessments are normalised to $100 \%$. Given is the percentage of reduction compared to baseline value for all patients (SD). P values are a Student's test between the differences before the course and after the course

\begin{tabular}{llll}
\hline Week & Before treatment & Immediately after treatment & 4 weeks after cessation of treatment \\
\hline Swollen joints & $14.7(3.9)$ & $-30 \%(30 \%)(\mathrm{P}=0.03)$ & $-20 \%(40 \%)(\mathrm{P}=0.15)$ \\
Tender joints & $11.9(6.5)$ & $-40 \%(40 \%)(\mathrm{P}=0.03)$ & $-40 \%(30 \%)(\mathrm{P}=0.01)$ \\
Restricted motion & $17.8(8.9)$ & $-50 \%(20 \%)(\mathrm{P}=0.003)$ & $-30 \%(30 \%)(\mathrm{P}=0.03)$ \\
Physician global assessment & & $-40 \%(40 \%)(\mathrm{P}=0.04)$ & $-60 \%(30 \%)(\mathrm{P}=0.01)$ \\
“Grip strength" & $18.5(12)$ & $+30 \%(40 \%)(\mathrm{P}=0.2)$ & $+60 \%(10 \%)(\mathrm{P}=0.2)$ \\
HAQ & $1.46(0.7)$ & $-10 \%(40 \%)(\mathrm{P}=0.9)$ & $-10 \%(70 \%)(\mathrm{P}=0.9)$ \\
Patient global assessment & & $-20 \%(40 \%)(\mathrm{P}=0.8)$ & $-40 \%(40 \%)(\mathrm{P}=0.1)$ \\
Morning stiffness & & $-10 \%(40 \%)(\mathrm{P}=0.9)$ & $-40 \%(40 \%)(\mathrm{P}=0.3)$ \\
20\% improvement criteria & 0 & $44 \%$ & $55 \%$ \\
\hline
\end{tabular}

Table 3 Changes in laboratory variables

\begin{tabular}{|c|c|c|c|c|c|c|}
\hline & \multicolumn{5}{|l|}{ Week No } & \multirow{2}{*}{ ANOVA test $I$} \\
\hline & 0 & 4 & 8 & 12 & 16 & \\
\hline$\overline{\text { CRP }}$ & $51(36)$ & $70(27)$ & $67(43)$ & $45(38)$ & $50(37)$ & 0.09 \\
\hline ESR & $70(32)$ & $78(30)$ & $68(30)$ & $58(33)$ & $58(32)$ & 0.45 \\
\hline $\mathrm{Hb}$ & $7.5(1.1)$ & $7.0(1.1)$ & $7.0(1.1)$ & $7.2(1.1)$ & $7.5(1)$ & 0.002 \\
\hline Albumin & 43.6 (3) & $41(3.6)$ & $40.5(3.0)$ & $45(9.5)$ & $43(3)$ & 0.22 \\
\hline Thrombocytes & $412(155)$ & $470(214)$ & $420(145)$ & 411 (194) & $372(145)$ & 0.05 \\
\hline Cortisol & $0.39(0.14)$ & $0.49(0.11)$ & $0.48(0.18)$ & $0.41(0.09)$ & $0.38(9)$ & 0.4 \\
\hline Free control & $20(9)$ & $23(5.6)$ & $25(12)$ & $18.5(5)$ & $18(10)$ & 0.7 \\
\hline II-6 & $11.8(6.1)^{\star}$ & $14.3(12.6)$ & $20.3(30.3)$ & $22(36)$ & $6.8(4.4)$ & 0.44 \\
\hline TNF & $9.1(17.5)$ & $7.8(16.6)$ & $6.2(11.3)$ & $5.3(7.8)$ & $5.3(9.5)$ & 0.38 \\
\hline
\end{tabular}

To assess the specificity of pentoxifylline/ thalidomide treatment for reducing TNF production, several other proinflammatory cytokines (IL-6, IL-12) and anti-inflammatory cytokines (IL-10) were measured in the whole blood culture supernatants. Table 1 shows that the production capacity for IL-6 was not affected, nor was the production of $\mathrm{IL}-12$ or IL-10 (table 3).

ANTIRHEUMATIC EFFECTS OF

THALIDOMIDE/PENTOXIFYLLINE

Of the nine patients who completed the study, five fulfilled the $-20 \%$ improvement criteria of the ACR core set of outcome measures at the end of the treatment. If individual indices were analysed the swollen joint count, tender joint count, restricted motion, and disease assessment by the physician improved significantly (table 2). ESR and CRP did not change during treatment (table 3 ). Since the hypothesis guiding this trial was a putative relation between inhibition of production capacity of TNF and antiarthritic effects the correlation was tested between the clinical response and decrease in TNF production capacity. Such a correlation was not observed. Also no correlation was observed between the decrease in TNF production capacity and reduction in swollen joint count (correlation -0.57) tender joint count (correlation 0.8), restricted motion (correlation 0.18), grip strength (correlation 0.39 ), or disease assessment judgements $(-0.76)$.

CHANGES IN ACUTE PHASE PROTEINS DURING PENTOXIFYLLINE/THALIDOMIDE TREATMENT

Table 3 shows laboratory variables during treatment. The trend in the laboratory variables was an increase in acute phase reactants. Some of the variables changed significantly, such as haemoglobin and thrombocytes, whereas others changed non- significantly (C reactive protein, ESR, IL-6, and albumin). These changes might be caused by an effect of the medication, for example, on the liver or a direct effect on cytokine production. The IL-6 concentration in plasma was not significantly increased during treatment. No significant correlations were found between the changes in production capacity of IL-6, IL-10, or IL-12 and the differences in concentrations of acute phase proteins before and during treatment.

The hypothalamic-pituitary axis is independently activated by the inflammatory cytokines $\mathrm{TNF} \alpha$, IL-1, and IL-6. ${ }^{14}$ During pentoxifylline/thalidomide the cortisol levels were increased, although not significantly. Finally, TNF concentrations in plasma were measured. Table 3 shows that the amounts of TNF in plasma were low and variable. No significant changes could be measured during the course of the study.

\section{Discussion}

This study showed that the combination pentoxifylline and thalidomide is rather toxic. Symptoms related to side effects were observed in the majority of the patients. The incidence of side effects was similar to that in other studies except for a higher incidence of xerostomia. ${ }^{113}$ The efficacy of simultaneous treatment with pentoxifylline and thalidomide in reducing TNF production was observed during treatment. This inhibition was specific, since production of IL-6, IL-10, and IL-12 was not affected.

The clinical effects of thalidomide/ pentoxifylline were such that $55 \%$ of the patients reached the $20 \%$ improvement criterion. This could be due to a placebo effect, regression to the mean, or a real effect. The absence of a correlation between clinical effect 
and the effect on TNF production does not suggest that the decrease in TNF producing capacity is causally related to reduction in disease activity, for example the swollen joint count. Moreover, the changes in the plasma IL-6, thrombocyte count, C reactive protein, ESR, albumin, haemoglobin, and cortisol were not suggestive of a reduction in TNF production in the arthritic joints. To clarify the issue of the clinical efficacy of pentoxifylline/ thalidomide in rheumatoid arthritis a placebo controlled trial is necessary.

The relation between the reduction of endotoxin induced TNF production measured ex vivo and a possibly reduced TNF production in the arthritic joint or an antiarthritic effect is uncertain. In this study no such relations were found. In another study of pentoxifylline in rheumatoid arthritis, seven out of 16 patients improved but there was also no observed correlation between clinical improvement and reduction in TNF production. ${ }^{7}$. This lack of correlation can be explained by sample size or might suggest that inhibition in the endotoxin induced TNF production is not relevant for the inflammatory process in rheumatoid arthritis. This interpretation contrasts with the beneficial effects of phosphodiesterase inhibitors in murine models of arthritis in which inhibition of endotoxin induced TNF production occurred simultaneously with antiarthritic effects. In numerous clinical situations pentoxifylline is used to reduce TNF mediated effects. However, no data are available showing that a reduction in TNF production affects the production of acute phase proteins. This phase I study was initiated on the basis that continuous production of TNF in the arthritic joints could be inhibited in a similar way to the TNF produced in endotoxin stimulated whole blood cultures. However, the lack of association between reduction in TNF production in endotoxin stimulated whole blood cultures and laboratory variables reflecting joint inflammation or clinical improvement brings the value of this substitute index of pharmacological efficacy into question. A large trial in which clinical efficacy in rheumatoid arthritis is measured simultaneously with whole blood cultures should be performed to validate or reject the use of whole blood cultures as a substitute index of pharmacological efficacy.

A Elouali, L van Rensen, J J Scoog, F Ouwerkerk, A Hardeman, and $\mathrm{H}$ Vermaat are acknowledged for their enthusiastic cooperation. D Lentjes is acknowledged for measurement of steroid hormones. T W J Huizinga is supported by a fellowship steroid hormones. T W J Huizinga is supported by a fellowship of the Royal Dutch Academy of Arts and Sciences. W Buurman,
University of Maastricht, The Netherlands, is acknowledged for University of Maastricht, The
providing the TNF ELISAs.

1 Elliott MJ, Maini RN, Feldmann M, Kalden JR, Antoni C, Smolen JS, et al. Randomised double-blind comparison of chimeric monoclonal antibody to tumour necrosis factor alpha (cA2) versus placebo in rheumatoid arthritis. Lancet 1994;344:1105-10.

2 Rankin ECC, Choy EHS, Kassimos D, Kingsley GH, Sopwith AM, Isenberg DA, et al. The therapeutic effect of an engineered anti-TNF antibody (CDP571) in rheumatoid arthritis. Br $\mathcal{F}$ Rheumatol 1995;34:334-43.

3 Han J, Thompson P, Beutler B. Dexamethasone and Pentoxifylline inhibit endotoxin-induced cachectin/TNF synthesis at separate points in the signaling pathway. $\mathcal{F} \operatorname{Exp}$ Med 1990;172:391-4.

4 Bianco JA, Appelbaum FR, Nemunaitis J, Almgren J, Andrews F, Kettner P, et al. Phase I-II trial of pentoxifylline for the prevention of transplant- related toxicities following bone marrow transplantation. Blood 1991; 78:1205-11.

5 Cid MC, Kleinman HK, Grant DS, Schnaper HW, Favei AS, Hofman GS. Estradiol enhances leukocyte binding to tumor necrosis factor (TNF)-stimulated endothelial cells via an increase in TNF-induced adhesion molecules E-selectin, intercellular adhesion molecule type 1, and E-selectin, intercellular adhesion molecule type 1, and
vascular cell adhesion molecule type $1 . \mathcal{F}$ Clin Invest 1994; vascular cell

6 Diperri G, Diperri IG, Monteiro GB, Bonora S, Hennig C, Cassatella M, et al. Pentoxifylline as a supportive agent in the treatment of cerebral malaria in children. $\mathcal{F}$ Infect $D i$ 1995;171:1317-22.

7 Maksymowych WP, Avinazubieta A, Luong MH, Russel AS. An open study of pentoxifylline in the treatment of severe refractory rheumatoid arthritis. $\mathfrak{f}$ Rheumatol 1995 ; 22:625-9.

8 Sampaio EP, Sarno EN, Galilly R, Cohn ZA, Kaplan G. Thalidomide selectively inhibits tumor necrosis factor alpha production by stimulated human monocytes. $\mathcal{F}$ Exp Med 1991;173:699-703.

9 Moreira AL, Sampaio EP, Zmuidzinas A, Frindt P, Smith KA, Kaplan G. Thalidomide exerts its inhibitory action on tumor necrosis factor alpha by enhancing mRNA degradation. $\mathcal{F} \operatorname{Exp} M e d$ 1993;177:1675-80.

10 Tramontana JM, Utaipat U, Molloy A, Akarasewi P, Burroughs $M$. Thalidomide treatment reduces tumor necrosis factor alpha production and enhances weight gain necrosis factor alpha production and enhances weight gain in patients with

11 Parker PM, Chao N, Nademanee A, O'Donnell MR, Schmidt GM, Snyder DS, et al. Thalidomide as salvage therapy for chronic graft-versus-host disease. Blood 1995;86:3604-9.

12 Gutierrez-Rodriguez O, Starusta-Bacal $P$, GutierrezMontes O. Treatment of refractory rheumatoid arthritisthe thalidomide experience. $\mathcal{f}$ Rheumatol 1989;16:159-63.

13 Revuz J, Guillaume J, Janier M, Hans P, Marchand C Souteyrand $\mathrm{P}$, et al. Crossover study of thalidomide vs placebo in severe recurrent aphthous stomatitis. Arch Dermacebo in severe recurr.

14 Chrousos GP. Seminars in medicine of the Beth Israel Hospital, Boston: the hypothalamic-pituitary-adrenal axis and immune-mediated inflammation. N Engl f Med 1995 332:1351-62. 